\title{
Endemic invasive amoebiasis in northern Australia
}

IN OCTOBER 2000, a 10-year-old Aboriginal boy from the Darwin region of the Northern Territory was referred to hospital with a 24-hour history of abdominal pain, initially generalised, but then localising to the right iliac fossa. The pain was accompanied by occasional vomiting, but no fever or diarrhoea was noted. At laparotomy, a gangrenous, unruptured appendix was removed. Postoperatively, the patient made a good recovery. Neither he nor any family members had travelled outside the Northern Territory.

Histological sections of the surgical specimen showed changes typical of acute suppurative appendicitis. Closer examination, however, revealed numerous round-to-oval structures resembling trophozoites (see Box). When the possibility of invasive amoebiasis was raised, staining of the section with Entamoeba histolytica-specific sera confirmed the diagnosis. E. histolytica serology was negative.

\section{Discussion}

E. histolytica is a protozoan parasite of humans that causes infectious colitis and amoebic liver abscess. It has recently been recognised that invasive disease is caused exclusively by the species $E$. histolytica, while the morphologically identical species $E$. dispar exists in the colonic lumen as a harmless saprophyte. $^{1}$

Patients with invasive amoebiasis living in Australia and other developed countries generally acquire the infection outside their country in a region where the pathogenic species is known to be endemic. While cases of amoebiasis in Australians who have not travelled overseas have been reported, ${ }^{2-6}$ all except one pre-date the recognition of pathogenic and non-pathogenic species, and in none was the primary source identified.

Isolated appendicitis due to E. histolytica is a rare condition. Even in regions where the organism is endemic, it causes less than $1 \%$ of cases of appendicitis. ${ }^{7}$

The diagnostic method of choice for amoebic colitis is testing for parasite antigen in stools. ${ }^{1}$ In this case, for the first time, the species-specific antibody normally used in the stool antigen test was adapted for immunohistochemistry and confirmed $E$. histolytica infection. Possible alternative methods for confirming the diagnosis include the polymerase chain reaction (PCR) or detection of $E$. histolytica-specific antibodies or parasite antigen in patient serum. ${ }^{1}$ However, PCR is not readily available outside research laboratories.
The sensitivity of the $E$. histolytica antibody test in intestinal disease is $52 \%$, a likely explanation for the negative serological test.

It is curious that no outbreaks of the readily identified and much more common manifestations of this infection, namely amoebic colitis and amoebic liver abscess, have been recently reported in northern Australia.

The potential public health significance of a case of proven autochthonous infection in an Australian population is reinforced by its high transmissibility in settings where hygiene may be suboptimal. ${ }^{8}$ Furthermore, there is a well documented possibility of prolonged latency between infection and the development of invasive disease. ${ }^{1}$ Both these factors support the merit of selective screening and appropriate treatment of at-risk contacts if invasive disease is detected. The use of new diagnostic techniques, such as the adapted staining method we used, will enable a more accurate determination of the endemicity of $E$. histolytica in Australia.

James S McCarthy, * David Peacock ${ }^{\dagger}$ Kevin P Trown, ${ }^{\ddagger}$ Patrick Bade ${ }^{\S}$ William A Petri Jr," Bart J Currie**

${ }^{\star}$ Associate Professor, Australian Centre for International and Tropical Health and Nutrition, University of Queensland, Royal Brisbane Hospital Herston, QLD. +Public Health Physician, Centre for Disease Control Territory Health Services, Darwin, NT; $¥$ Pathologist Western Diagnostic Pathology, Darwin, NT

SSurgeon, Darwin Private Hospital, Darwin, NT

\section{Entamoeba histolytica in an inflamed} appendix

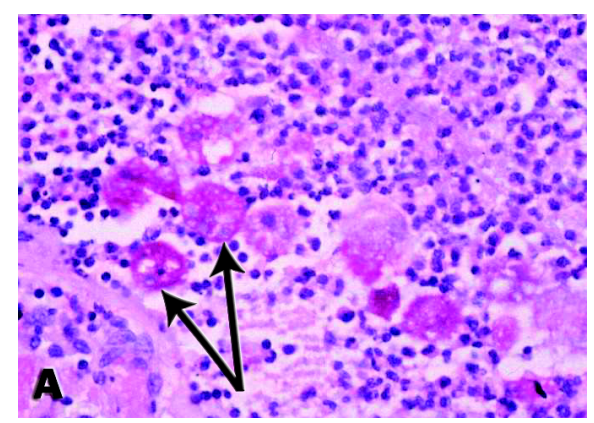

A: Periodic acid-Schiff stain, showing trophozoites (arrowed) within an inflammatory infiltrate.

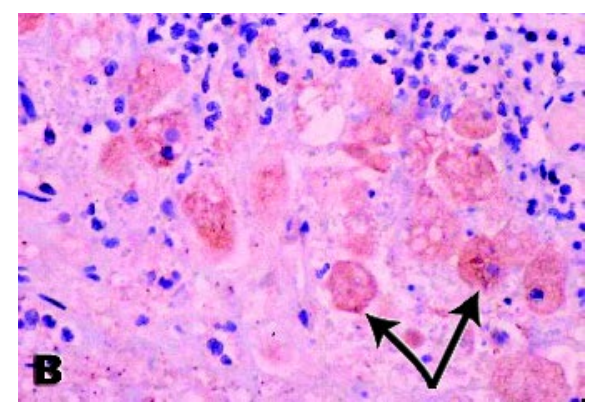

B: Immunoperoxidase stain of trophozoites (arrowed) using E. histolytica-specific sera. Note the surrounding necrotic tissue. Professor, Departments of Medicine, Microbiology and Pathology, University of Virginia, Charlottesville Virginia, USA; ** Professor, Menzies School of Health Research and Northern Territory Clinical School Royal Darwin Hospital, Darwin, NT J.McCarthy@sph.uq.edu.au

Acknowledgements: We would like to thank Dr Lionel Crompton and Dr Rhian Kenrick of Fred's Pass Medical Centre, Humpty Doo, NT; and Dr Ross Glancy and Mr Geoff Swan of the Pathology Department, Fremantle Hospital, WA. WAP Jr received financial support from $\mathrm{NIH}$ grant Al-43596.

. Petri WA Jr, Singh U. Diagnosis and management of amebiasis. Clin Infect Dis 1999. 29: 1117-1125.

2. Lancaster HO. An amoebic survey of Australian soldiers during the Second World War. Med J Aust 1953; 1: 552-553.

3. Norcott TC. Experience with the Entamoeba histolyt ica fluorescent antibody test in Western Australia. Med J Aust 1973; 2: 21-22.

4. Ravenscroft PJ, Douglas JB, Emmerson BY, et al. Intestinal amoebiasis and amoebic liver abscess in Brisbane, 1956 to 1973. Clinical syndromes and the results of medical and surgical management. Med $J$ Aust 1975; 1: 551-555.

5. Weinmann AJ, Spelman DW, Spicer WJ. Indigenous invasive amoebiasis in Australia. Aust N Z J Surg 1992; 62: 235-237.

6. Zurauskas JP, McBride WH. Case of amoebic liver abscess: prolonged latency or acquired in Australia? Intern Med J 2001; 31: 565-566.

7. Bhaskar KV Malik AK, Sharma SC et al. Isolated amebic appendicitis: a pathologic rarity. Am J Gastroenterol 1988; 83: 1188-1189.

8. Haque R, Ali IK, Sack RB, et al. Amebiasis and mucosal IgA antibody against the Entamoeba histoIytica adherence lectin in Bangladeshi children. $J$ Infect Dis 2001; 183: 1787-1793.

(Received 21 Feb, accepted 7 Jun 2002) 